\title{
Attention to People Like You: A Proposal Regarding Neuroendocrine Effects on Linguistic Variation
}

\author{
Míša Hejná ${ }^{1,}$, Lauren Ackerman², \& Joel C. Wallenberg ${ }^{3}$
}

${ }^{1}$ Department of English, Faculty of Arts, Aarhus University, Denmark

2 School of Education, Communication, \& Language Science, Faculty of Humanities \& Social Sciences, Newcastle University, United Kingdom

${ }^{3}$ School of English Literature, Language, \& Linguistics, Faculty of Humanities \& Social Sciences, Newcastle University, United Kingdom

*Corresponding author: misa.hejna@cc.au.dk

MH: https:/ / orcid.org/0000-0002-9328-2603

LA: https:// orcid.org/0000-0002-3223-8641

JCW: https://orcid.org/0000-0002-1837-6765

Although the literature on language change has often replicated and discussed a pattern in which female speakers lead in changes that occur below the level of awareness, there is no consensus on why this pattern should arise. Interestingly, recent findings in endocrinology show that differences in prenatal testosterone exposure can impact learning patterns. In the light of these findings, we first present preliminary results consistent with the hypothesis that a biological factor, prenatal exposure to androgens, can have a small, continuous biasing effect on linguistic variation, namely the variable duration of pre-aspiration conditioned by voiceless obstruents in Tyneside English. Second, we propose an explanatory model in which the biological factor-prenatal testosterone exposure-creates subtle bias in how speakers learn linguistic variants and suggest that some reported sex effects are derivative. This model is compatible with the high tendency for females to lead in language change from below (Labov 1990: 206).

Keywords: pre-aspiration; prenatal testosterone exposure; sound change from below; gender/sex differences; Tyneside English

\section{Introduction}

When discussing sound change, Labov (1966 [2006]: 209) distinguishes sound change from above and sound change from below awareness, or consciousness.

Editor: $\quad$ Kleanthes K. Grohmann, University of Cyprus, Cyprus 
Sound change from above refers to the situation in which speakers associate an innovative variant within their community with prestige and in which explicit comments on such variants by the speakers of the relevant community can be found (see e.g., Labov 1966, who reports that higher rates of postvocalic /r/ correlate with higher prestige in New York City English). Sound change from below then refers to those sound changes where the adoption of the innovative feature is not associated with prestige and where the speakers of the community do not comment on the innovation explicitly (see e.g., Baker et al. 2011 for sretraction, whereby $/ \mathrm{s} /$ in words such as street sounds more like a $/ \mathrm{J} /$; and Lawson et al. 2008, who report de-rhoticisation of postvocalic /r/ in Scottish English as change from below). ${ }^{1}$ It is widely reported in quantitative sociolinguistics that there are measurable differences in the frequencies at which speakers use linguistic variables cross-linguistically (e.g., Labov 2001). This is particularly true of ongoing sound changes below the level of consciousness (as defined in Labov 1966 [2006]: 209), where female speakers have been found to "lead" (e.g., Labov 1990, 2001). That is, controlling for other social variables, female speakers tend to use variants that are more progressive within their communities (for continuous sound changes, such as vowel fronting-e.g., Labov, Rosenfelder and Fruehwald 2013, Przedlacka 2001: 47, Hinrichs, Bohmann and Gorman 2013), and also tend to use the new variant more frequently than male counterparts (for categorical variants, such as the presence of post-vocalic $/ \mathrm{r} /$ in New York City English-Labov 1966, 1994, 2001). In other words, during the spread of a change from below through a community, the intraspeaker variation of female speakers tends to be a bit different than that of male speakers: on average, a female speaker can be expected to produce more advanced variants more often than a male speaker within the same generation.

This paper focuses on sound change from below and the mechanisms whereby women often end up leading in this type of change. We may wonder what might underlie the observation that women tend to learn or adopt new variants at a higher rate than men. Two major suggestions have been put forward. On the one hand, Labov (1990: 219) suggests that women are most frequently the primary caregivers in most communities, but that boys learn to distance themselves from female norms during their childhood, while girls model their caregivers more faithfully. On the other hand, Eckert (2011) has suggested that women are more likely to engage in "social engineering" and "symbolic domination" than men, due to the setup of the gender roles in their communities, which makes them more likely to be linguistic innovators. ${ }^{2}$ Despite these two major attempts to explain why female speakers lead in sound change from below, there is nevertheless no current consensus as to why this is the case. Furthermore,

1 For the purposes of this paper, we are not concerned with the difference between 'awareness' and 'consciousness'. We suspect that these terms could be used to differentiate different intermediate orders of indexicality, but for our purposes, anything that a speaker or community could explicitly identify and discuss as being variant will count as 'above' and anything that goes unremarked upon will count as 'below'.

2 There is also the proposal according to which male speakers are also agentive in that they purposely differentiate themselves from female speakers (e.g., Trudgill 1972). However, for our purposes this falls within a generally agentive group of explanations. 
proposals predicated on the agentivity of speakers in a "linguistic marketplace" are hard-pressed to explain an effect that specifically applies to changes from below the level of consciousness, which do not confer prestige, and specifically not to changes from above the level of consciousness. In particular, when referring to sound change from below, we are concerned with the concept generally accepted within the field of the theory of sound change and sociolinguistics: namely, the process whereby an innovative variant spreads throughout a community, this community comprising multiple generations. We do not focus on the emergence of innovative variants.

Therefore, this paper proposes an account in which subtle biases in learning and cognition contribute to the observed patterns in community change from below (i.e. across speakers), by influencing how speakers represent the interspeaker variation in their internal systems. Particularly, neurological development (which modulates learning) is affected by exposure to prenatal testosterone (see review in Balthazart 2011), which is sexually dimorphic. We suggest that it is a small effect of prenatal $\mathrm{T}$ on learning that accounts for the reported differences between men and women, rather than necessarily their active construction of different social roles. Our proposed model is compatible with the neuroendocrinological literature and the sociolinguistic literature, and may make substantial contributions to our understanding of change from below.

Models including any role for neurological/biological factors have rarely been considered seriously (e.g., Labov 2001: 291), and when they have been, they have run the risk of gender essentialism. One of the reasons behind this avoidance of biological factors is due to the methodological challenges of engaging with what these factors entail. For instance, regarding research in biological ageing, establishing the appropriate methods is relatively recent (e.g., Belsky et al. 2015) and many methods available are invasive or otherwise prohibitive. Therefore, these methods are less likely to be accessible to linguists for reasons including ethics of invasive or medical procedures, financial limitations, and importantly the relevant interdisciplinary expertise. However, there are also a number of methods that are non-invasive and financially viable (e.g., Belsky et al. 2015, Wong and Hines 2016). Although engaging even with non-invasive methods is still a cross-disciplinary challenge, this is not insurmountable, as we will show in this paper.

We propose a potential mechanism whereby female speakers typically end up as leaders in sound change from below in the absence of prestige factors as potential motivators. We suggest that one source of this difference could be individual differences in learning from human models, modulated by biological factors. More specifically, it may be the case that the way the prenatal hormone milieu differentially influences development of the brain, the organizing effects of sex hormones on the brain, creates underlying biases in social learning that are different from speaker to speaker, and broadly different between the sexes. These individual biases in social attentiveness then result in individual differences in how speakers sample the linguistic variation they observe in their community and form an internal model for that variation in their linguistic competence (see e.g., Yang 2000, 2002, for a more full discussion of the acquisition of intraspeaker variation as a function of sampling community variation). While this could 
broadly result in the differentiated use of linguistic variants by men and women during change in progress, we suggest that the male/female effect is derivative from a gradient biological property, and the underlying effect is driven by a continuous bias regarding attentiveness to social models in one's age cohort. This proposal is based on two types of evidence: research from other fields, including neuroendocrinology and developmental psychology, and an exploratory case study of our own. By engaging with the methodology used in neuroendocrinology and developmental psychology, our study first shows that it is possible to extend the methodology to the field of linguistics, and also presents important results suggesting that the organizing effects attributed to prenatal exposure to sex hormones lead to a small but consistent bias in perception and learning that underlies some apparently sex-dimorphic language use. We suggest that the continuous biological factor of prenatal exposure to androgens has a gradient effect on the distribution of a linguistic variant. Our case study looks into preaspiration before voiceless obstruents in Tyneside English (see Section 2.2), a phenomenon which has been reported to be on the rise in this variety of English, with Watt \& Allen (2003) showing that younger individuals produce preaspiration more frequently. We test this hypothesis by investigating individual differences in the duration of pre-aspiration within a single sex cohort. We use the ratio of the length of the index finger to the ring finger as a continuous biological proxy for early life androgen exposure (2D:4D, see section 2.1 for more details), a measure which has been correlationally linked to the testosterone/estradiol ratio in amniotic fluid in humans (Lutchmaya et al. 2004), and experimentally linked to testosterone exposure in rats (Talarovičová et al. 2009). We propose a model, supported by the empirical observations we report here, in which social learning interacts with biological factors, together contributing to observed patterns of variation during an unconscious sound change in progress (i.e. "change from below").

The field of behavioral neuroendocrinology has shown that the prenatal hormonal milieu of developing organisms bears a potentially causal relation to various adult behaviors in both humans and non-humans (Hines et al. 2004, Hines 2006, Balthazart \& Adkins-Regan 2002, Balthazart 2011). Prenatal exposure to testosterone in humans influences various childhood behaviors, including the later adoption of stereotypically gendered behaviors (sometimes referred to as "sex roles", "gender roles", or "gender" more broadly, e.g., Ackerman 2019): For prenatal T on childhood behavior see Pasterski et al. (2005), Auyeung et al. (2009), Cohen-Bendahan et al. (2005), and Hines et al. (2016); for social learning of genderrelated labels for objects as in toys that are "for girls" or "for boys" see Hines et al. (2016); for infant eye-contact Lutchmaya et al. (2002); and for lateralisation of certain cognitive functions Liu et al. (2017) and Manson (2008). We would suggest that what unites these various behavioral results is a relationship between prenatal $\mathrm{T}$ exposure and a sensitivity to the social models an individual learns from. It could be that higher pre-natal testosterone exposure $(\mathrm{T})$ leads to less sensitive social learning, with learners sampling a wide variety of behaviors they see around them, whereas lower pre-natal $\mathrm{T}$ leads to greater pickiness on the part of learners, causing them to focus on people closest to them in various characteristics (e.g., sex, age, etc). Thus, we propose that these behaviors indicate an underlying 
mechanism that could also influence the pattern of uptake and spread of new linguistic variants below the level of awareness, apparently led by women due not to purely social factors but also neuroendocrinological organizational effects. We predict that proxies for prenatal testosterone exposure levels, here the 2D:4D digit ratio, will have a significant effect on the amount speakers use a more innovative variant.

Sections 2 and 3 present our exploratory experiment that looks into whether prenatal testosterone exposure correlates with an innovative variant within a single-sex cohort. We then outline how a biosocial model may work in detail in Section 4, where we also flag areas of this proposal that require further investigation.

\section{Materials and Methods}

\subsection{Quantifying Prenatal Androgen Level}

While it is difficult to access information about the early-life hormonal milieu in non-clinical human populations, indirect biomarkers do exist. The ratio of the second digit (index finger) to the fourth digit (ring finger), herein referred to as 2D:4D, has been shown in infants to reflect their mothers' amniotic ratios of testosterone to estradiol (Lutchmaya et al. 2004), which makes it a useful albeit noisy proxy for retrospectively measuring prenatal androgen exposure in humans (Cohen-Bendahan et al. 2005, Wong and Hines 2016). Though this biomarker cannot be ethically validated in humans experimentally, it has been shown that experimental manipulation of prenatal testosterone in rat fetuses results in a smaller 2D:4D claw-length ratio after the rats are born (Talarovičová et al. 2009). The effect is more pronounced on the right hand for both rodents (Talarovičová et al. 2009) and humans (Cohen-Bendahan et al. 2005), and right-hand 2D:4D has been shown to predict a level of conformity to aspects of stereotypical gender roles within a single-sex cohort (Atkinson et al. 2017, Brown et al. 2002). ${ }^{3}$

In order to calculate individuals' 2D:4D ratio in our study, participants were asked to place their right hand palm-down on a Doxie portable scanner, producing a portable networks graphic (PNG) image at 600 dpi resolution. The length of the second and fourth digits were then measured from the basal crease to the tip of the finger with digital calipers available in GNU Image Manipulation Program (GIMP). This procedure was chosen because digital measurement of scanned images has been shown to be the most accurate and consistent method of calculating 2D:4D ratio (Allaway et al. 2009).

\subsection{Identifying and Quantifying Pre-aspiration}

Pre-aspiration is defined here as a period of voiceless, primarily glottal friction which occurs in the sequences of sonorants and phonetically voiceless obstruents, represented as a superscript $\mathrm{h}$ in words such as mass $\left[\mathrm{ma}^{\mathrm{h}} \mathrm{s}\right]$ and $\mathrm{mat}^{\mathrm{t}}\left[\mathrm{ma}^{\mathrm{h}} \mathrm{t}^{\mathrm{s}}\right]$ (Nance

3 This is far from a claim that the sex roles or gendered behaviors themselves are innate. Rather, it is only an observation that this measure bears a relationship to conformity. 


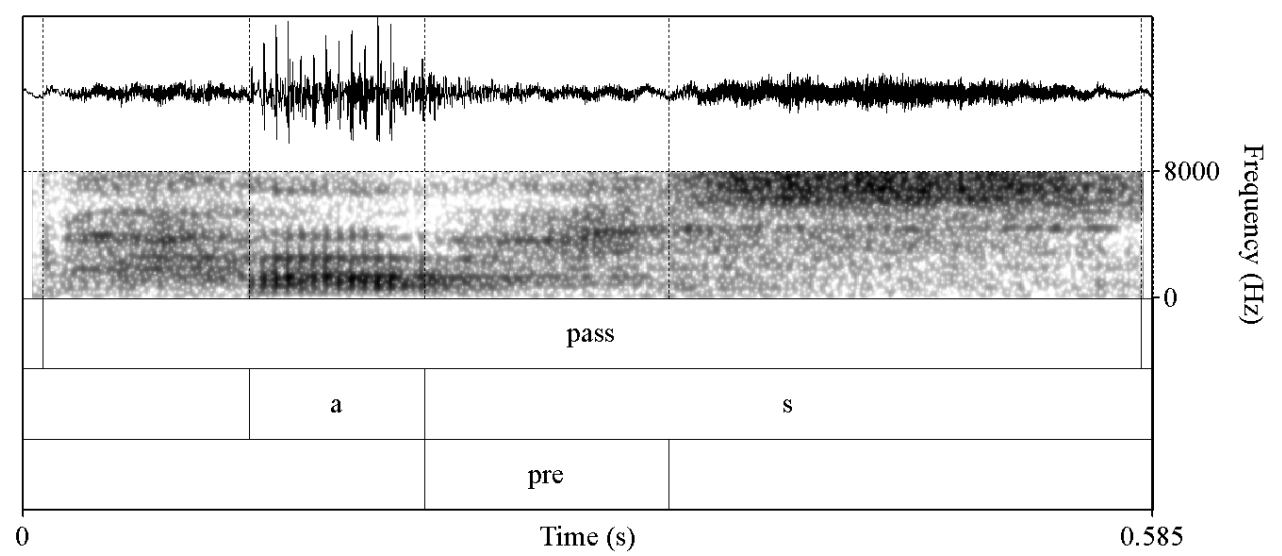

Figure 1: Identification of pre-aspiration in a fricative context.

and Stuart-Smith 2013, Hejná and Scanlon 2015). We identified the presence of pre-aspiration and quantified its duration as described below (see Hejná 2016 for more details).

In the plosive environment (e.g., mat), the left boundary of pre-aspiration was determined by the absence of voicing associated with the vowel and the right boundary by the absence of pre-aspiration friction. In the fricative environment (e.g., mass), the identification of the left boundary was the same as in the plosive context. The right boundary, however, could not be determined on the basis of the lack of friction because fricatives are by definition articulated as turbulent sounds that lack closure, just like pre-aspiration. We therefore used more detailed spectral criteria for the right boundary. While $/ \mathrm{J} /$ and $/ \mathrm{s} /$ show a concentration of high-intensity energy at higher frequencies, glottal friction shows low-intensity energy spread across the frequencies, as shown in Figure 1. The highlighted portion in the figure delimits pre-aspiration in the word pass. The voicing associated with the vowel stops at the left-hand edge of pre-aspiration, and the high-frequency band of energy associated with the friction of /s/ begins at the right-hand edge of pre-aspiration.

In the cases of $/ \theta /$ and $/ f /$, the criterion based on the distinct centers of energies is not always sufficiently reliable because the two oral fricatives are associated with a fairly even spread of energies across the frequencies as well, in contrast to / s / and / $/$ / Fry 1982). In these cases, it was the structure of the bands of higher energy in the pre-aspiration friction (i.e., formant structure) that distinguished it from the friction of the two oral fricatives: pre-aspiration formant structure tends to closely mirror that of the local vowel, which is distinct from the typical patterns of energy distribution in oral fricatives.

Only positive cases of pre-aspiration (i.e., durations greater than $0 \mathrm{~ms}$ ) were included in the analyses. Pre-aspiration duration was normalized as a natural logarithm of the percentage of the overall word duration in order to account for potential speaking rate differences and prosodic lengthening effects. 


\subsubsection{Descriptive Statistics}

The data comprise 1,533 tokens with pre-aspiration, ranging from 13 to 157 tokens per speaker, with an average of 69.7 tokens and a median of 55 tokens. The words analyzed contain the following structures: $\mathrm{CVC}(\mathrm{C})(\mathrm{C})$ (as in pass, past, lasts) and $\mathrm{CVC}(\mathrm{C}) \mathrm{V}$ (as in messy, misty); in which the first syllable is always stressed and the second syllable is not if the word has more than a single syllable. Our analysis only includes pre-aspiration in lexically stressed contexts, since this is where preaspiration has been reported to be produced most frequently (e.g., Hejná 2015: Chap. 3, and the references therein). With these structures, we coded for the variables of vowel quality (in the stressed syllable; 15 levels, see Figure 2) and the immediately adjacent, pre-aspiration inducing obstruent (eight levels, see Figure $3)$.

These phonetic environments of the tokens were not balanced across speakers because this cannot be controlled for in naturalistic interview data intended to produce casual speech. Thus, the preceding vowel phoneme and the place of articulation of the local consonant are included as factors in the statistical analyses.

In addition, we also coded for the linguistic context immediately following the word analysed; namely, we distinguished following words as (1) those starting with a vowel (e.g., ...cat and $d o g)$; (2) those that started with a consonant (e.g., ...cat running); and (3) pauses (e.g., ...cat.). This was done because in phenomena such as / $\mathrm{t} /$-flapping, flapping applies in sequences such as get it and ate it. Since we did not know based on the literature available whether this may also affect pre-aspiration, it was deemed prudent to code for this potential factor. Finally, the

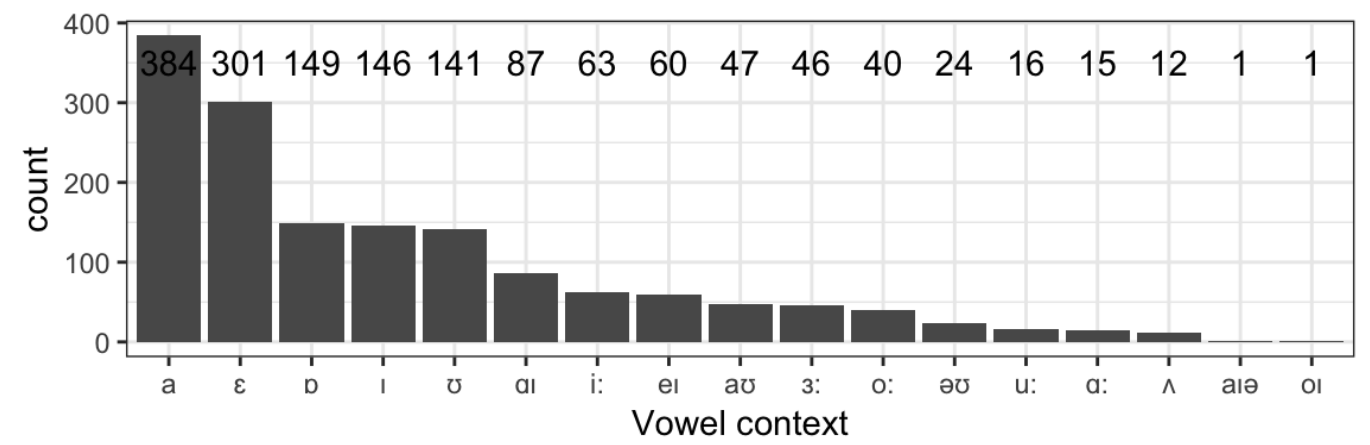

Figure 2: Distribution of vowel phonemes preceding pre-aspiration in our dataset.

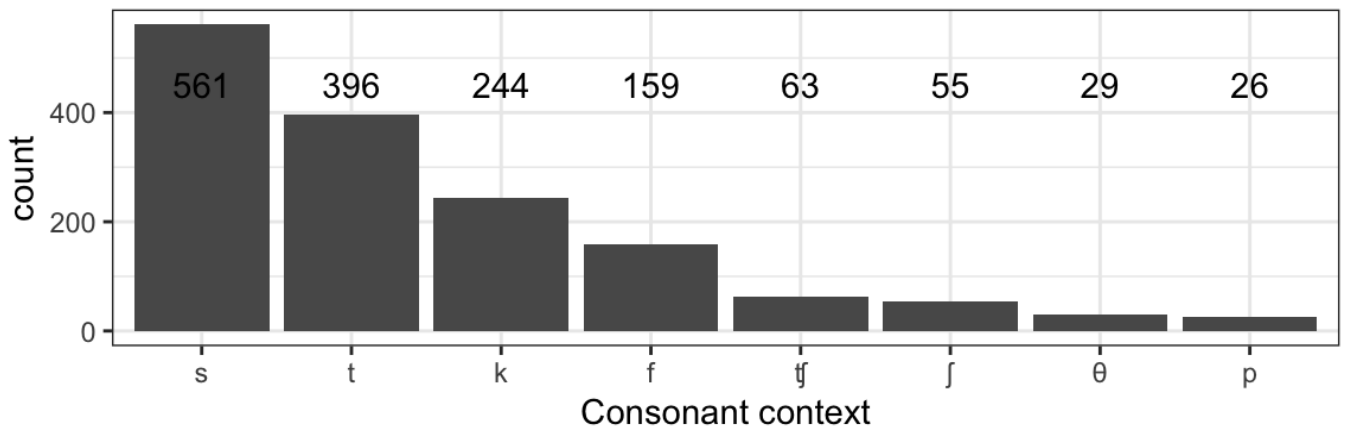

Figure 3: Distribution of obstruent phonemes inducing pre-aspiration in our dataset. 
pause context was included because of domain final lengthening effects as well. Tokens with pre-aspiration were followed by a consonant in 506 cases, by a pause or silence in 598 cases, and by a vowel in 429 cases.

\subsection{Participants}

Twenty-two participants identifying themselves to researchers as women/female were recruited from the Tyneside area and interviewed using standard sociolinguistic procedures (Tagliamonte 2006). They ranged in age from 22 to 45 years of age, with a mean age of $30.9(\mathrm{SD}=8.7)$ and median age of 29.5. Each person was asked a series of prompts in order to elicit a naturalistic speech sample, which averaged approximately one hour in duration. All participants gave signed consent, and were provided with information about the study, investigators, and university ethics procedures. Twenty of the participants were White, of European heritage. One participant was primarily of East Asian heritage, and one participant was primarily of South Asian heritage. As we did not have enough participants from different ethnicities to reliably model any potential differences by ethnicity (Manning et al. 2007), we did not include ethnicity as a separate variable in the analytical models.

\subsection{Recording and Data Processing}

Interviews were recorded one-to-one on a Zoom H4n Handy Recorder in a quiet room, using the built-in microphone. The data were sampled at $44.1 \mathrm{kHz}$ and stored in .wav format. Interview transcriptions were force-aligned to the recorded sound files using FAVE-align (Rosenfelder et al. 2014), then manually checked for accuracy in the relevant words. The phonetic segments preceding and following the pre-aspiration were extracted, along with the lexical environment and syllabic stress. Pre-aspiration was annotated manually. Ambiguous cases were excluded from the analyses. Datasets used for the analyses above are publicly available (see Data Availability statement).

\section{Results}

To assess the contribution of prenatal exposure to androgens to the usage of preaspiration, we compared a series of linear mixed effects regression models based on the principle of parsimonious model design (Bates et al. 2018). The contribution of the 2D:4D ratio was the independent variable of interest ('logDigit'), and the variation in pre-aspiration duration as a proportion of word duration ('logProp') was the dependent variable. Other fixed effects were speaker age, the interviewer (two levels), the vowel context (see Figure 2), the consonantal segment triggering pre-aspiration (see Figure 3), the metrical foot position (medial, as in better, or final, as in bet), and the following segment (consonant, vowel, or pause). A random intercept for speaker was included but the structure of random slopes was limited by the naturalistic nature of the data. Since the factors that are logically able to vary randomly by speaker contain a large number of categorical levels (e.g., triggering consonant environment, vowel context), these factors were too 
imbalanced to include in the analysis as interactions. This analysis was performed using the lme4 package (Bates et al. 2015) in $\mathrm{R}$ (R Core Team) using the following formula: lmer (logProp logDigit + age + interviewer + vowelContext + triggerConsonant + footPosition + followingseg + (1 | speaker), data=data, REML=FALSE). To calculate the contribution of the relevant factor to the overall model fit, a chi-square test was used to compare this full model to a nested subset model which had the single relevant term removed.

The linear mixed effects regression analysis revealed a significant main effect of 2D:4D digit ratio, with higher ratios (corresponding to lower androgen exposure) correlated with longer proportional pre-aspiration durations $(\beta=1.31$, $\left.\mathrm{SE}=.47, \chi^{2}(1)=6.6, \mathrm{p}=0.010\right)$. This small but significant effect is confirmed by a Pearson's product-moment correlation test $(\mathrm{t}=6.2, \mathrm{df}=1531, \mathrm{p}<0.0001$; CI 95\% $=0.11-0.20 ; \mathrm{r}=0.16$ ). These results suggest that speakers who were exposed to lower levels of androgens during prenatal development also produce longer preaspiration in Tyneside English, as illustrated in Figure 4. What Figure 4 shows is that the effect of $2 \mathrm{D}: 4 \mathrm{D}$ ratio on the log of proportional pre-aspiration durations is small; yet, it is nevertheless statistically significant. This is consistent with the hypothesis that prenatal hormone organizing effects could contribute to the

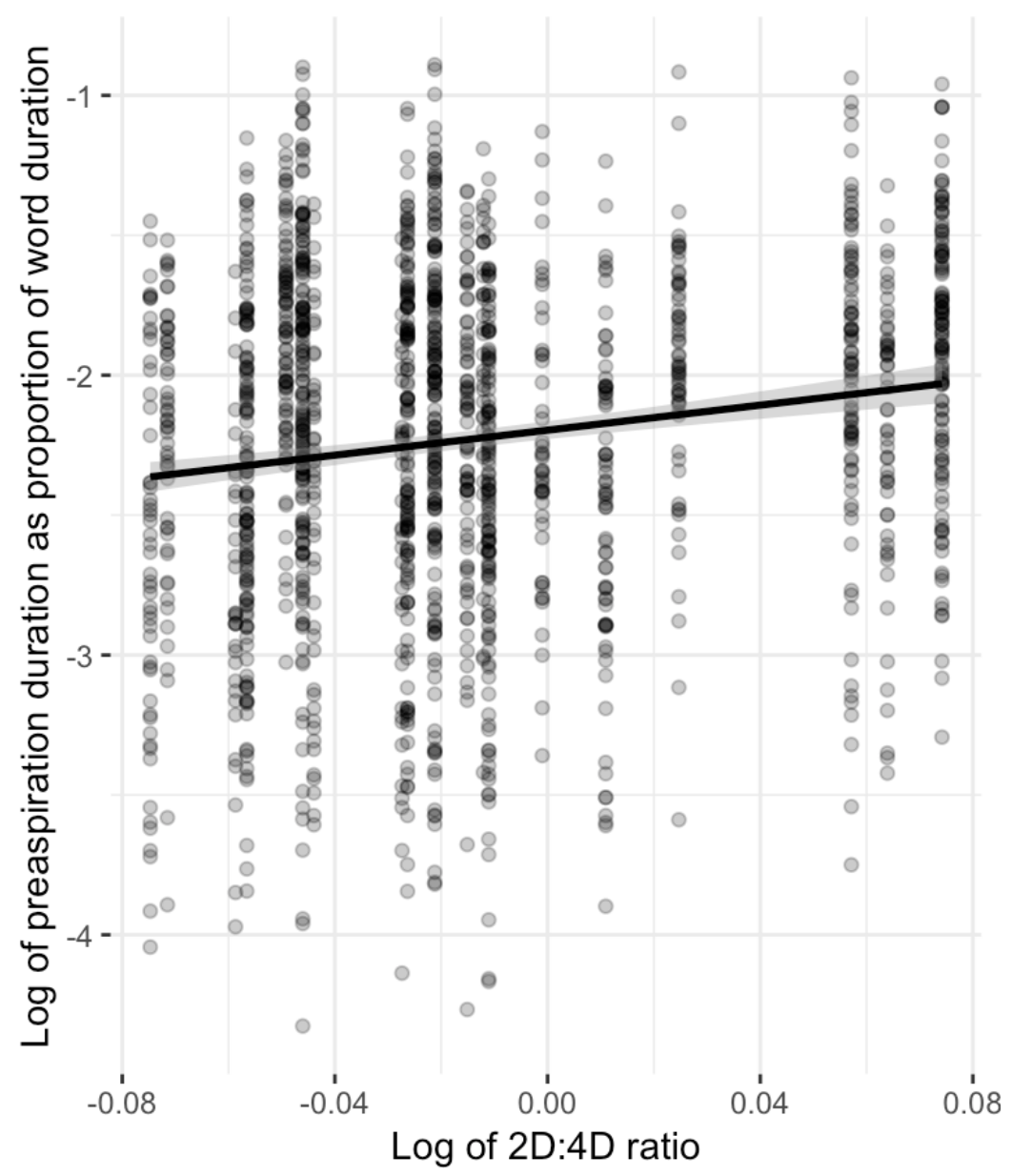

Figure 4: Lower prenatal androgens correlate with more advanced variant. 
observed differences in language variation from below the level of consciousness, which have typically been attributed to social effects of gender.

Speaker age did not significantly contribute to this model $(\beta<0.01, \mathrm{SE}<$ $\left.0.01, \chi^{2}(1)=2.1, p=0.14\right)$. Age was expected to contribute to the variation of a sound change in progress, since such changes typically are detectable in apparent time; that is, people of different ages demonstrate different stages of the sound change when contemporaneous speech samples are compared. It is possible that the sound change in question had been completed at the time of interviews, thus no change in apparent time is detectable. It is also possible that our sample of speakers is (relatively) homogeneous and uniform in age, leading to a lack of power in this particular dimension. However, the significant correlation between 2D:4D ratio and pre-aspiration suggests that this line of research may prove fruitful in future investigations. ${ }^{4}$ We now turn to discussing a biosocial mechanism whereby sound changes from below often emerge as led by female speakers in their communities.

\section{Discussion}

\subsection{A biosocial model}

Our study provided evidence that, even within a single-sex sample, prenatal testosterone exposure has an inverse association with the duration of preaspiration. In other words, lower levels of prenatal testosterone correlate with longer pre-aspiration duration. Since this parallels observations made in the wider literature which are attributed to sex or gender differences, we suggest that the often observed sex effect may actually stem from a more basic effect, as sex cohorts will typically also differ in their hormone profiles. We propose a possible mechanism that can account for the previously observed sex differences in studies of sound change in light of the observed individual inter-speaker variation. The suggestion is to some extent in line with Labov (1990), who proposes that the explanation may lie in the learning conditions of future language users, who tend to be exposed more frequently to females due to their primary caregivers being female. However, while we agree that learning mechanisms may be key, our proposal diverges from Labov's in several ways. Firstly, we propose that the learning strategies involved vary not only across but also within sex cohorts. Secondly, this can be independent of whether the primary caregiver is female or male; rather, differences in learning strategies can be related to variation in prenatal testosterone exposure. Regarding Eckert's (2011) line of explanation, ours differs from hers in that we exclude agency on the part of the speakeradopter as the sole driving mechanism. Crucially, however, the model we propose is assumed to co-exist with possible agentive factors discussed by Eckert, linked to group membership.

Uncontroversially, individuals differentially learn and reproduce linguistic features undergoing population-level change, leading to subtle inter-speaker

The variable of interviewer (with two levels: one female from Newcastle, one male from San Francisco) did not show any effects on pre-aspiration duration. 


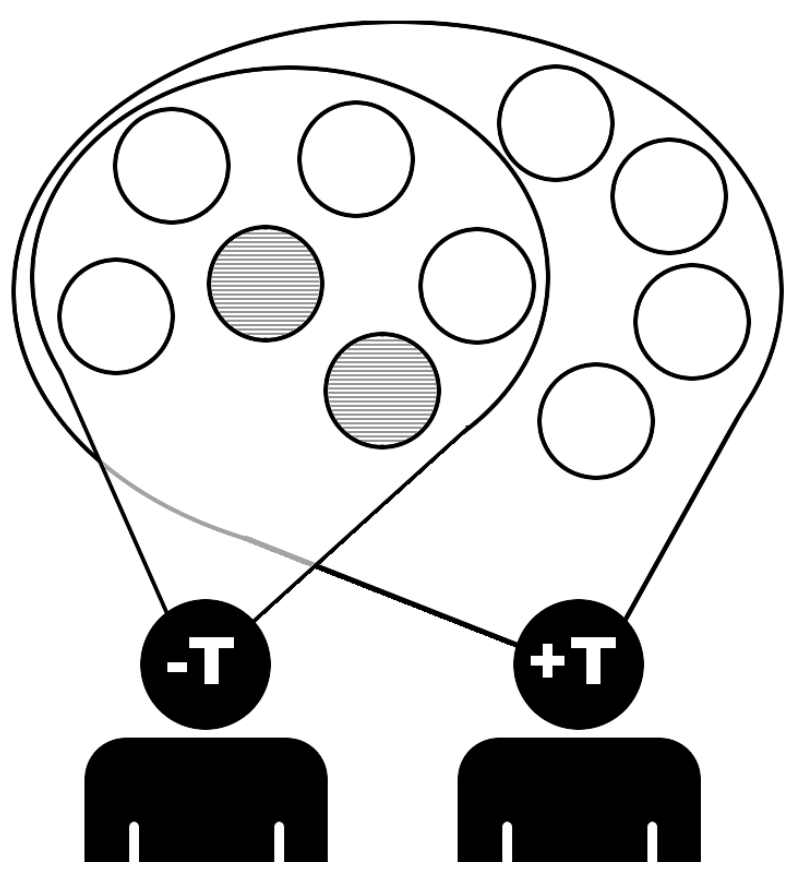

Figure 5: A biosocial model of attention during language acquisition.

individual differences in linguistic behavior. Building on the research related to social learning patterns and endocrine organizing effects we mentioned above in Introduction, we posit a biosocial model that takes seriously these influences on early-life development, and suggests a mechanism for the sexually dimorphic behavior during language change that is missing from the extant literature (e.g., Labov 1990, Eckert 2011). We hypothesise that the differences in infant eye-contact Lutchmaya et al (2002) report between higher prenatal $\mathrm{T}$ infants and lower prenatal T infants is an indication of generally better social attentiveness in lower prenatal $\mathrm{T}$ individuals, throughout life. If that is the case, then individuals exposed to higher levels of prenatal testosterone $(+\mathrm{T})$ would be less attentive to appropriate adult social models than individuals exposed to lower levels of prenatal testosterone $(-\mathrm{T})$. We refer to this variation in attentiveness as "attentional specificity", which means being sensitive to all potentially socially salient characteristics of people in the population, including their ages.

Figure 5 illustrates such an effect of attentional specificity on sound change in the following way: In this illustration, circles correspond to individuals in a population of speakers. Shaded circles indicate speakers with a high frequency of a new linguistic variant or a more advanced versions of a continuous variant: suppose the actuation of some new change from below has already occurred, and a new variant has begun its spread. The left-hand individual $(-\mathrm{T}$, lower testosterone exposure) attends more to social models who are more similar in terms of social factors such as age, thus samples the speech of only a subset of the population which they are exposed to. In this subset, the new linguistic variant is heard in one third of the observations. The right-hand individual $(+\mathrm{T}$, higher testosterone exposure) is less selective in their attentiveness to social models, and so samples randomly from the entire population. This speaker hears the new 
variant proportionally less often because only one fifth of their sample observations contain it. If speakers store the frequencies of variants they hear in language acquisition and use these to form an internal model of the variation and thus their target frequencies of the variants for production (as extensively argued in Yang 2000, 2002, among others), the -T speaker will then produce a higher frequency of the new linguistic variant than the $+\mathrm{T}$ speaker. Thus, individual variation in social attention, as biased by prenatal hormone organizing effects, influences the proportion of tokens of a new variant that an individual samples during language acquisition and learning. Because attentional specificity influences who in the population an individual attends to and learns from, individuals with lower prenatal exposure to testosterone are more likely to learn from those in their peer group (i.e., with whom they are closest in age). As children learn their first language, these individuals will attend to a younger subset of the speaker population to which they are exposed. One can think of this as a model of how learners sample from all the community productions their hear, and this sample is a given learner's primary linguistic data (see the example sampling scenarios in Figure 5 and Figure 6).

In Figure 6, this process is illustrated in more detail than in Figure 5 by four variants of an individual that differ in prenatal exposure to testosterone, ranging from least $(--\mathrm{T})$ to most $(++\mathrm{T})$; note that these categories are for illustrative purposes, and amount of pre-natal $\mathrm{T}$ exposure is, in reality, a continuous variable. In individuals with typical hormonal profiles, we may assume females with lower prenatal testosterone exposure correspond to --T, females with somewhat higher prenatal testosterone exposure correspond to $-\mathrm{T}$, males with somewhat lower prenatal testosterone exposure correspond to $+\mathrm{T}$, and males with higher prenatal testosterone exposure correspond to $++\mathrm{T}$. All individuals are within the same age cohort (Cohort A), with Cohort B being slightly older than A, Cohort C older than $B$, and Cohort D representing the rest of the population at large. Circles filled in with grey backgrounds indicate speakers producing a variant that is spread mostly evenly across the population. Circles with horizontal stripes represent speakers producing a variant that has been innovated in younger cohorts, thus is concentrated in younger cohorts at this point in time. Table 1 describes the differ-

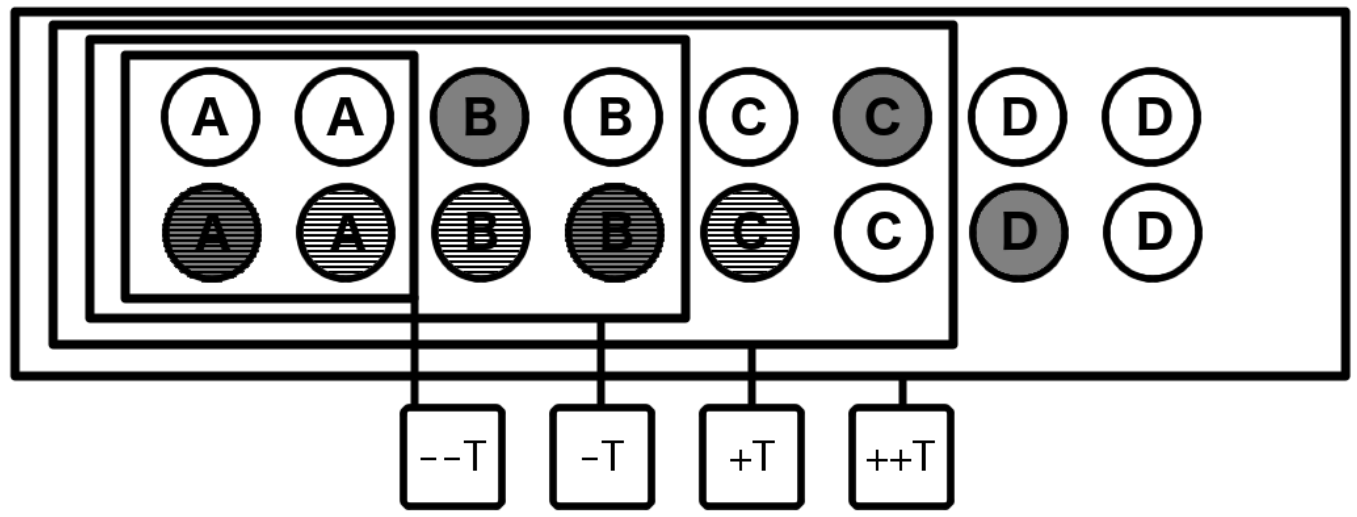

Figure 6: Example of how this model would play out in a (simplistic but illustrative) population. An interactive version of this model is available at: https://lmackerman.shinyapps.io/aplymodel201904 (as of 6 May 2019). 


\begin{tabular}{|c|c|c|c|c|}
\hline & \multicolumn{2}{|c|}{$\begin{array}{c}\text { Grey circles (evenly spread } \\
\text { throughout population) }\end{array}$} & \multicolumn{2}{c|}{$\begin{array}{c}\text { Striped circles (concentrated in } \\
\text { younger cohorts) }\end{array}$} \\
\hline Individual & $\begin{array}{c}\text { Number of social } \\
\text { models producing } \\
\text { new variant }\end{array}$ & $\begin{array}{c}\text { Proportion of social } \\
\text { models producing } \\
\text { new variant }\end{array}$ & $\begin{array}{c}\text { Number of social } \\
\text { models producing } \\
\text { new variant }\end{array}$ & $\begin{array}{c}\text { Proportion of social } \\
\text { models producing } \\
\text { new variant }\end{array}$ \\
\hline$++\mathrm{T}$ & 5 & $31.3 \%$ & 5 & $31.3 \%$ \\
\hline$+\mathrm{T}$ & 4 & $33.3 \%$ & 5 & $41.7 \%$ \\
\hline$-\mathrm{T}$ & 3 & $37.5 \%$ & 4 & $50 \%$ \\
\hline$--\mathrm{T}$ & 1 & $25 \%$ & 2 & $50 \%$ \\
\hline
\end{tabular}

Table 1: Rate of exposure based on individual variation in attention to social models, i.e., differential attention to speech produced by similar age-cohort models modulated by prenatal testosterone exposure.

ential rates at which each of the four listeners would be exposed to the two types of features, as relevant for the example shown in Figure 6.

From this illustration (Figure 6), individuals are exposed to the "grey" feature at similar rates, with slight variation between the $--\mathrm{T}$ and $-\mathrm{T}$ individuals due to a quirk of the distribution in the population. If we assume that the $--\mathrm{T}$ and $-\mathrm{T}$ individuals are both female and the $+\mathrm{T}$ and $++\mathrm{T}$ individuals are male, this pattern of exposure would result in little to no variation in uptake across the population. In contrast, the "striped" feature that skews young is adopted at higher rates by the female individuals than the male individuals; the new variant spreads over time in both men and women, but females will be consistently ahead of males at every time point in the spread. Given a binary categorization by sex, this could appear to be a property that varies categorically. However, when individuals are assessed on a gradient scale, we can see that this pattern is predicted by prenatal exposure to testosterone and the individuals' social network in tandem. ${ }^{5}$

The more selectively attentive individuals, as they acquire language in early life, sample speech from a subset of the population that produces more of the new variant which is concentrated in younger speakers (who match in age with the learners). If less exposure to prenatal testosterone is associated with more attentional specificity, female speakers will, on average, show larger amounts of new linguistic variants undergoing change below awareness than male speakers of the same age throughout the inter-generational spread of a new linguistic variant.

Crucially, however, there are four important things that need to be added. First, because the distribution of an innovative variant is not uniform across a speech community, nor is-presumably—that of individuals with specific levels of prenatal testosterone exposure, it cannot always be the case that the most innovative variants will be found with highest rate of application / most advanced realization in the following order: females with relatively lower testosterone exposure $(--\mathrm{T})>$ females with relatively higher testosterone exposure $(-\mathrm{T})>$ males with relatively lower testosterone exposure $(+\mathrm{T})>$ males with relatively higher testosterone exposure $(++\mathrm{T})$. Second, changes from below show clear apparent time distributions: we are more likely to find innovations in

Here we have only focused on age-related aspects of social networks, and in a rather simplistic way in comparison to highly complex real-life situations. The proposed model needs to be developed to deal with social networks in a more in-depth ways in future. 
younger age cohorts (e.g., Tagliamonte 2016). This fact alone makes it more likely that younger cohorts are exposed to those speakers with the innovative features in age stratified cultures.

Thirdly, the mechanism we propose is additional to the bias for younger speakers to be the source of innovative features. In other words, the prenatal testosterone exposure bias adds to that of innovative features typically arising in children and peaking in adolescent cohorts. These individuals with higher attentional specificity will then learn and produce more of the new variant than those in their age cohort with lower attentional specificity, since these latter speakers will have sampled more broadly from the relevant population. As the proportion of the population using the new variant increases, those with lower attentional specificity will become more likely to pick it up, but they will do so at a lower proportion than the high attentional specificity speakers. This lag is due to dilution of the new variant in a differentially larger population sample for individuals with lower attentional specificity. Note that the mechanism we propose here for the sex-differential lag is independent of the mechanism that is responsible for the incrementation of the change from below. It is, for instance, entirely compatible with a "momentum-based" model for incrementation (Bermúdez-Otero, to appear). In that scenario, adolescents increment the frequency or advancedness of a new variant in line with their representation of an age vector for that variant in the population. Our mechanism would simply mean that the represented age vector is systematically a little different in $-\mathrm{T}$ vs $+\mathrm{T}$ individuals, because they have estimated it on the basis of a slightly different sampling of the population. Their incrementation based on this age vector will then also have slightly different outcomes, even if $-\mathrm{T}$ and $+\mathrm{T}$ speakers increment by the same amount.

In what follows, we also discuss two important methodological factors that may have bearing on the model we suggest and which need to be taken into account in future research.

\section{2. $\quad$ Further considerations}

\subsubsection{Laryngeal physiology}

One potential confound in assessing the plausibility of the attentional specificity mechanism is the possibility that prenatal testosterone exposure could have a direct effect on the physiology of the vocal apparatus, thus producing the observed pre-aspiration pattern. In other words, we must address the concern that the effect reported may be attributable to laryngeal structure differences rather than those related to brain structures involved in learning and attention. This is especially the case since the larynx is generally seen as a secondary sexual organ (e.g., Abitbol et al. 1989, Amir \& Biron-Shental 2004, Collins \& Missing 2003, Hall 1995, Henton \& Bladon 1985), with its sexual characteristics resulting from circulating (activational) hormone effects (see e.g., Raj et al. 2008, Wadnerkar et al. 2006, Whiteside et al. 2004, for the effects of menstrual cycle on voice quality and laryngeal properties of consonants), although establishing whether and where there are hormonal receptors in the laryngeal structures is controversial 
(Nacci et al. 2011). Interestingly, Fouquet et al. (2016), who conducted a real-time study of $\mathrm{f} 0$ of 10 male speakers at 7-year intervals starting at 7 and ending at 56 years of age, found that intra-sex differences are apparent already at the age of 7 and persist to adulthood. The authors suggest this may be due to prenatal androgen exposure differences (2016: 7) rather than those of circulating hormones that lead to vocal differences across the two sexes during puberty; however, information related to prenatal testosterone exposure was not available to the research team. Ferdezi et al. (2011) have presented a study which looked into whether there is a correlation between their male speaker's f0 and their 2D:4D of either the left or the right hand: no correlation was found, suggesting that the laryngeal structures are not affected by prenatal testosterone exposure.

Additionally, it is indicative that the effect of 2D:4D holds for preaspiration, but not for the other laryngeal phenomena that we quantified in addition to pre-aspiration, namely local breathiness and creaky voice (see e.g., Hejná 2015: Chap. 3, for local breathiness, and Keating, Garellek \& Kreiman 2015 for creaky voice, respectively). As shown in Figure 7, while the correlation between 2D:4D and pre-aspiration duration is significant, no such correlations are observed for breathiness or creakiness in our data.

This suggests that the effect is not due to prenatal testosterone exposure on the laryngeal structures. On the other hand, different laryngeal phenomena rely on different laryngeal gestures and it is not known which laryngeal structures should be targeted by prenatal testosterone exposure. For this reason, future work should include a range of linguistic phenomena that rely on different parts of the vocal tract to conclusively rule out effects of prenatal testosterone exposure on the laryngeal structures rather than on general learning mechanisms associated with brain structure differences affecting linguistic production.

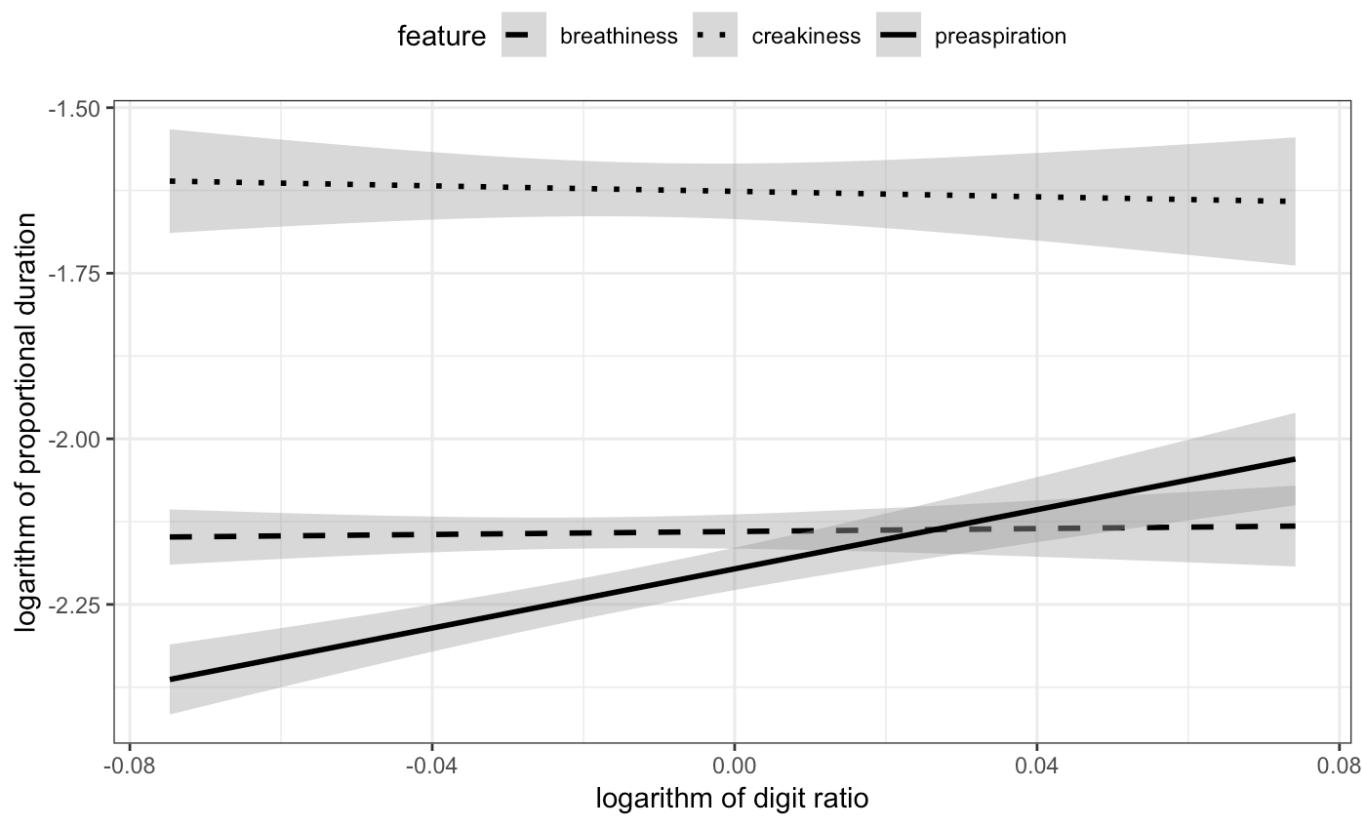

Figure 7: No correlation between 2D:4D and breathiness and creaky voice. Breathiness: $r=0.0078$; creakiness: $r=-0.0150$, pre-aspiration: $r=0.1555$. 


\subsection{2. $2 D: 4 D$}

Another caveat of this study is the use of the 2D:4D ratio, which is controversial in its use as a biomarker for human exposure to prenatal testosterone. The previous studies on the relationship between testosterone and 2D:4D in humans and rats, respectively, do not examine precisely the same biological relationships, thus must be regarded carefully (Lutchmaya et al. 2004, Talarovičová et al. 2009). Additionally, there are other differences in the timing of sexual organizing effects in rats and humans, and the way sex hormones enter the brain may be different in the two species (Balthazart 2011). See also reviews of 2D:4D analyses, which note various inconsistencies in reported studies linking 2D:4D and behavior (Cohen-Bendahan et al. 2005, Wong \& Hines 2016). Future work might circumvent these issues by using other biomarker proxies, potentially including spontaneous or click-evoked otoacoustic emissions (McFadden 2002, McFadden \& Pasanen 1998, 1999), or ideally, by accessing longitudinal data gathered from a population whose prenatal testosterone levels were measured.

\section{Conclusion}

In this study, we demonstrate that a potential biomarker for prenatal androgen exposure (2D:4D) predicts the duration of pre-aspiration, a sound change in progress in Tyneside English (albeit with a weak correlation). Since prenatal testosterone has also been shown to co-vary with some aspects of later social behaviors, we suggest a mechanism whereby social attentiveness relates to the propagation of linguistic variants over a number of generations. In particular, since lower levels of prenatal testosterone predict more advanced linguistic variants during a change, we suggest that people who were prenatally exposed to lower levels of testosterone will be more likely to learn new linguistic variants during a sound change in progress, due to a small bias in the social models they attend to, although future modelling should confirm how frequently this arises in a range of realistic social network setups vis-à-vis linguistic innovations. The small bias in attending to social models like themselves affects the way different individuals sample the population of speakers they are exposed to.

While these results are not yet fully conclusive, they do indicate that biological factors may have a role in explaining some patterns of language change observed throughout the sociolinguistic literature. For changes below the level of awareness, this small but persistent influence of prenatal testosterone organizing effects could explain the apparently sex-correlated observations in the literature. We hope that the suggestive results reported here provide a starting point for further investigation into how small, individual differences in biological development influence social attention, learning styles, and linguistic learning.

\section{Data Availability}

The data used for analysis in this article are freely available from the Open Science Framework: http://doi.org/10.17605/OSF.IO/P5NKA 


\section{Acknowledgements}

We would like to thank Claire Cochrane for her immense help with data collection and participant recruitment. We are also grateful to the Centre for Behaviour and Evolution seminar audience at Newcastle University. This work was, in part, supported by a Wellcome Trust Capital Award to Newcastle University (grant number 092504). All errors are, of course, our own.

\section{References}

Ackerman, Lauren. 2019. Syntactic and cognitive issues in investigating gendered coreference. Glossa: A Journal of General Linguistics 4(1), 117. doi:10.5334/ gigl.721

Allaway, Heather C., Terri G. Bloski, Roger A. Pierson \& Marla E. Lujan. 2009. Digit ratios (2D:4D) determined by computer-assisted analysis are more reliable than those using physical measurements, photocopies, and printed scans. American Journal of Human Biology 21(3), 365-370.

Abitbol, Jean, Jean de Brux, Ginette Millot, Marie-Françoise Masson, Odile Languille Mimoun, Helene Pau \& Beatrice Abitbol. 1989. Does a hormonal vocal cord cycle exist in women? Study of Vocal Premenstrual Syndrome in voice performers by videostroboscopy-glottography and cytology on 38 women. Journal of Voice 3(2), 157-162.

Amir, Ofer \& Tal Biron-Shental. 2004. The impact of hormonal fluctuations on female vocal folds. Current Opinion in Otolaryngology and Head and Neck Surgery 12(3), 180-184.

Atkinson, Beth M., Tom V. Smulders \& Joel C. Wallenberg. 2017. An endocrine basis for tomboy identity: The second-to-fourth digit ratio (2D:4D) in "tomboys". Psychoneuroendocrinology 79, 9-12.

Auyeung, Bonnie, Simon Baron-Cohen, Emma Ashwin, Rebecca Knickmeyer, Kevin Taylor, Gerald Hackett \& Melissa Hines. 2009. Fetal testosterone predicts sexually differentiated childhood behavior in girls and in boys. Psychological Science 20(2), 144-148.

Baker, Adam, Diana Archangeli \& Jeff Mielke. 2011. Variability in American English s-retraction suggests a solution to the actuation problem. Language Variation and Change 23(3), 347-374.

Balthazart, Jacques. 2011. The Biology of Homosexuality. Oxford: Oxford University Press.

Balthazart, Jacques \& Elizabeth Adkins-Regan. 2002. Sexual differentiation of brain and behavior in birds. Hormones, Brain and Behavior 4, 223-301.

Bates, Douglas, Reinhold Kliegl, Shravan Vasishth \& Harald Baayen. 2018. Parsimonious mixed models. arXiv:1506.04967.

Bates, Douglas, Martin Mächler, Ben B. Bolker \& Steve Walker. 2015. Fitting Linear Mixed-Effects Models Using lme4. Journal of Statistical Software 67(1), $1-48$.

Belsky, Daniel W., Avshalom Caspi, Renate Houts, Harvey J. Cohen, David L. Corcoran, Andrea Danese, HonaLee Harrington, Salomon Israel, Morgan E. Levine, Jonathan D. Schaefer, Karen Sugden, Ben Williams, Anatoli I. 
Yashin, Richie Poulton \& Terrie E. Moffitt. 2015. Quantification of biological aging in young adults. PNAS, 1-7.

Bermúdez-Otero, R. To appear. Individual differences and the explanation of sound change. In James Kirby, Lauren Hall-Lew \& Patrick Honeybone (eds.), Individuals, Communities, and Sound Change. Glossa special collection.

Brown, Windy M., Christopher J. Finn, Bradley M. Cooke \& S. Marc Breedlove. 2002. Differences in finger length ratios between self-identified "butch" and "femme" lesbians. Archives of Sexual Behavior 31(1), 123-127.

Cohen-Bendahan, Celina C. C., Cornelieke van de Beek \& Sheri A. Berenbaum. 2005. Prenatal sex hormone effects on child and adult sex-typed behavior: methods and findings. Neuroscience and Biobehavioral Reviews 29(2), 353-384.

Collins, Sarah A. \& Caroline Missing. 2003. Vocal and visual attractiveness are related in women. Animal Behaviour 65, 997-1004.

Eckert, Penelope. 2011. Language and power in the preadolescent heterosexual market. American Speech 86(1), 85-97.

Ferdezi, Camille, Jean-François Lemaître, Juan David Leongómez \& S. Craig Roberts. 2011. Digit ratio (2D:4D) predicts facial, but not voice or body odour, attractiveness in men. Proceedings of the Royal Society B 278(1724), 3551-3557.

Fouquet, Meddy, Katarzyna Pisanski, Nicolas Methevon \& David Reby. 2016. Seven and up: individual differences in male voice fundamental frequency emerge before puberty and remain stable throughout adulthood. Proceedings of the Royal Society B 3(10), 160395.

Fry, Dennis B. 1982. The Physics of Speech. (Cambridge Textbooks in Linguistics). Cambridge: Cambridge University Press.

Hall, Kira. 1995. Lip service on fantasy lines. In Kira Hall \& Mary Bucholtz (eds.), Gender Articulated: Language and the Socially Constructed Self, 183-226. New York: Routledge.

Hall, Kira \& Mary Bucholtz (eds.). 1995. Gender Articulated: Language and the Socially Constructed Self. New York: Routledge.

Hejná, Míša. 2015. Pre-aspiration in Welsh English: A case-study of Aberystwyth. Manchester, UK: University of Manchester dissertation.

Hejná, Míša. 2016. Pre-aspiration: manual on acoustic analyses 1.1. Available from: https:/ / ling.auf.net/lingbuzz/003184

Hejná, Míša \& Jane Scanlon. 2015. New laryngeal allophony in Manchester English. Proceedings of the 18th International Congress of Phonetic Sciences, Glasgow.

Henton, C. G. \& R. A. W. Bladon. 1985. Breathiness in normal female speech: inefficiency versus desirability. Language and Communication 5(3): 221-227.

Hines, Melissa. 2006. Prenatal testosterone and gender-related behaviour. European Journal of Endocrinology 155 (supplement 1), S115-S121.

Hines, Melissa, Charles Brook \& Gerard S. Conway. 2004. Androgen and psychosexual development: core gender identity, sexual orientation, and recalled childhood gender role behavior in women and men with congenital adrenal hyperplasia (CAH). Journal of Sex Research 41(1), 75-81.

Hines, Melissa, Vickie Pasterski, Debra Spencer, Sharon Neufeld, Praveetha Patalay, Peter Hindmarsh, Ieuan A. Hughes \& Carlo L. Acerini. 2016. 
Prenatal androgen exposure alters girls' responses to information indicating gender-appropriate behaviour. Philosophical Transactions of Royal Society B 371(1688), 20150125.

Hinrichs, Lars, Alex Bohmann \& Kyle Gorman. 2013. Real-time trends in the Texas English vowel system: F2 trajectory in GOOSE as an index of a variety's ongoing delocalization. Rice Working Papers in Linguistics 4, 1-12.

Keating, Patricia, Marc Garellek \& Jody Kreiman. 2015. Acoustic properties of different kinds of creaky voice. Proceedings of 18th International Congress of Phonetic Sciences, Glasgow.

Labov, William. 1966. The Social Stratification of English in New York City. Washington, D.C.: Center for Applied Linguistics.

Labov, William. 1990. The intersection of sex and social class in the course of linguistic change. Language Variation and Change 2, 205-254.

Labov, William. 1994. Principles of Linguistic Change. Volume 1: Internal Factors. Oxford: Blackwell.

Labov, William. 2001. Principles of Linguistic Change. Volume 2: Social Factors. Oxford: Blackwell.

Labov, William, Ingrid Rosenfelder \& Josef Fruehwald. 2013. One hundred years of sound change in Philadelphia: linear incrementation, reversal, and reanalysis. Language 89(1), 30-60.

Lawson, Eleanor, Jane Stuart-Smith \& James Scobbie. 2008. Articulatory insights into language variation and change: Preliminary findings from an ultrasound study of derhoticization in Scottish English. University of Pennsylvania Working Papers in Linguistics 4(2), 102-110.

Liu, Jinfeng, Dan Wang, Xiaoting Li \& Wang Ningyu. 2017. Association between sex and speech auditory brainstem responses in adults, and relationship to sex hormone levels. Medical Science Monitor 23, 2275-2283.

Lutchmaya, Svetlana, Simon Baron-Cohen \& Peter Raggatt. 2002. Foetal testosterone and eye contact in 12-month-old human infants. Infant Behavior and Development 25(3), 327-335.

Lutchmaya, Svetlana, Simon Baron-Cohen, Peter Raggatt, Rebecca Knickmeyer \& John T. Manning. 2004. 2nd to 4th digit ratios, fetal testosterone and estradiol. Early Human Development 77(1), 23-28.

Manning, John T., Andrew J. G. Churchill \& Michael Peters. 2007. The effects of sex, ethnicity, and sexual orientation on self-measured digit ratio (2D: 4D). Archives of Sexual Behavior 36(2), 223-233.

Manson, JoAnn E. 2008. Prenatal exposure to sex steroid hormones and behavioral/ cognitive outcomes. Metabolism 57, S16-S21.

McFadden, Dennis. 2002. Masculinization effects in the auditory system. Archives of Sexual Behavior 31(1), 99-111.

McFadden, Dennis \& Edward G. Pasanen. 1998. Comparison of the auditory systems of heterosexuals and homosexuals: Click-evoked otoacoustic emissions. Proceedings of the National Academy of Sciences 95(5), 2709-2713.

McFadden, Dennis \& Edward G. Pasanen. 1999. Spontaneous otoacoustic emissions in heterosexuals, homosexuals, and bisexuals. Journal of the Acoustical Society of America 105(4), 2403-2413. 
Nacci, Andrea, Bruno Fattori, Fabio Basolo, Maria E. Filice, Katia De Jeso, Luca Giovannini, Luca Muscatello, Fabio Matteucci \& Francesco Ursino. 2011. Sex hormone receptors in vocal fold tissue: a theory about the influence of sex hormones in the larynx. Folia Phoniatrica et Logopaedica 63, 77-82.

Nance, Claire \& Jane Stuart-Smith. 2013. Pre-aspiration and post-aspiration in Scottish Gaelic stop consonants. Journal of the International Phonetic Association 43(1), 129-152.

Pasterski, Vickie L., Mitchell E. Geffner, Caroline Brain, Peter Hindmarsh, Charles Brook \& Melissa Hines. 2005. Prenatal hormones and postnatal socialization by parents as determinants of male-typical toy play in girls with congenital adrenal hyperplasia. Child Development 76(1), 264-278.

Przedlacka, Joanna. 2001. Estuary English and RP: some recent findings. Studia Anglica Posnaniensia 36, 35-50.

R Core Team. 2016. R: A Language and Environment for Statistical Computing. Available from: https:// www.r-project.org/

Raj, Anoop, Bulbul Gupta, Anindita Chowdhury \& Shelly Chadha. 2008. A study of voice changes in various phases of menstrual cycle and in postmenopausal women. Journal of Voice 24(3), 363-368.

Rosenfelder, Ingrid, Josef Fruehwald, Keelan Evanini, Scott Seyfarth, Kyle Gorman, Hilary Prichard \& Jiahong Yuan. 2014. FAVE (Forced Alignment and Vowel Extraction) Suite Version 1.1.3.

Tagliamonte, Sali. 2006. Analysing Sociolinguistic Variation. Cambridge: Cambridge University Press.

Tagliamonte, Sali. 2016. Teen Talk. The Language of Adolescents. Cambridge: Cambridge University Press.

Talarovičová, Alžběta, Lucia Kršková \& Jana Blažeková. 2009. Testosterone enhancement during pregnancy influences the 2D:4D ratio and open field motor activity of rat siblings in adulthood. Hormones and Behavior 55(1), 235239.

Trudgill, Peter. 1972. Sex, covert prestige and linguistic change in the urban British English of Norwich. Language in Society 1, 179-195.

Watt, Dominic \& William Allen. 2003. Tyneside English. Journal of the International Phonetic Association 33(2), 267-271.

Wadnerkar, Meghana B., Patricia E. Cowell \& Sandra P. Whiteside. 2006. Speech across the menstrual cycle: a replication and extension study. Neuroscience Letters 408, 21-24.

Whiteside, Sandra P., Anna Hanson \& Patricia E. Cowell. 2004. Hormones and temporal components of speech: sex differences and effects of menstrual cyclicity on speech. Neuroscience Letters 367, 44-47.

Wong, Wang I. \& Melissa Hines. 2016. Interpreting digit ratio (2D:4D)-behavior correlations: 2D:4D sex difference, stability, and behavioral correlates and their replicability in young children. Hormones and Behavior 78, 86-94.

Yang, Charles. 2000. Internal and external forces in language change. Language Variation and Change 12(3), 231-250.

Yang, Charles. 2002. Knowledge and Learning in Natural Language. Oxford: Oxford University Press. 